\title{
Modelo de Markowitz y Modelo de Black-Litterman en la Optimización de Portafolios dle Inversión
}

\author{
Luis C. Franco-Arbeláez ${ }^{1}$ \\ Claudia T. Avendaño-Rúa ${ }^{2}$ \\ Haroldo Barbutín-Díaz ${ }^{3}$
}

\section{Resumen}

La optimización de portafolios de inversión es un aspecto central en el mundo financiero. El modelo de Markowitz ha logrado éxito a nivel teórico en el medio de las finanzas, en cuanto a la estructuración de portafolios y en la búsqueda de la diversificación implícita en el análisis de inversiones. Sin embargo, en la práctica, se presentan dificultades e inconvenientes, que han influido notoriamente en el poco éxito de su aplicación. En este artículo se hace un estudio reflexivo sobre las desventajas de este modelo en situaciones reales, y se presenta el modelo de Black-Litterman como alternativa metodológica que contribuye a neutralizar algunas de esas desventajas y permite maximizar el rendimiento esperado, generando un portafolio más eficiente, estable y diversificado.

\section{Palabras clave}

Markowitz, Black-Litterman, volatilidad, riesgo, gestión de portafolios, expectativas.

1 Programa de Ingeniería Financiera y de Negocios, INSTITUTO TECNOLÓGICO METROPOLITANO, luisfranco41446@apolo.itm.edu.co

2 Programa de Ingeniería Financiera y de Negocios, INSTITUTO TECNOLÓGICO METROPOLITANO, claudiaavendano0456@apolo.itm.edu.co

3 Programa de Ingeniería Financiera y de Negocios, INSTITUTO TECNOLÓGICO METROPOLITANO, haroldobarbutin7345@apolo.itm.edu.co

Fecha de recepción: 16 de Agosto de 2010

Fecha de aceptación: 23 de Febrero de 2011 


\section{Abstract}

The optimization of investment portfolios is a central aspect in the financial world. Markowitz's model has been successful on a theoretical level in the middle of finance, about the structuring of portfolios and the search of implicit diversify in the investment analysis. However, in practice, there are difficulties and disadvantages that has been a notable influence on the limited success of its implementation. In this article is done a reflective study of the disadvantages of this model in real situations, and presents the Black-Litterman model as an alternative methodology that helps to neutralize some of these disadvantages and maximizing the expected return, generating a more efficient, stable and diversified portfolio.

\section{Keywords}

Markowitz, Black-Litterman, volatility, risk, portfolio management, views. 


\section{INTRODUCCIÓN}

La investigación y el análisis de las teorías en el manejo del riesgo asociado a cualquier inversión son fundamentales para el desarrollo de nuevas ideas y aplicaciones en el área de las finanzas.

El modelo de Harry Markowitz, desde su origen en 1952, contribuyó a variados desarrollos y derivaciones, proporcionando el marco conceptual del manejo eficiente de un portafolio, maximizando la rentabilidad esperada y controlando el riesgo. Sin embargo, en la práctica no ha sido tan difundido, por sus reconocidas debilidades en contextos reales (Michaud, 1989).

El objetivo general del presente artículo consiste en realizar un estudio comparativo entre el modelo de Markowitz y el modelo de Black-Litterman, para determinar las ventajas relativas del segundo y obtener conclusiones pertinentes. Después de esta introducción, se hace una descripción detallada del modelo de Markowitz y sus desventajas más reconocidas. Luego se presenta el modelo de Black-Litterman, que permite corregir algunas de las falencias generadas en ese modelo; y finalmente se obtienen conclusiones.

\section{MODELO DE HARRY MARKOWITZ}

En 1952 el economista norteamericano Harry Markowitz, especialista en análisis de inversiones, publicó un artículo llamado "Portfolio Selection" en donde expone su teoría sobre cómo hallar la composición óptima de un portafolio de valores, maximizando la rentabilidad para un determinado nivel máximo de riesgo aceptable; o en forma alternativa, minimizar el riesgo para una rentabilidad mínima esperada.

\subsection{Hipótesis del Modelo de Markowitz}

El modelo de Markowitz parte de las siguientes hipótesis: a) El rendimiento de cualquier portafolio, es considerado una variable aleatoria, para la cual el inversionista estima una distribución de 
probabilidad para el periodo de estudio. El valor esperado de la variable aleatoria es utilizado para cuantificar la rentabilidad de la inversión; b) la varianza o la desviación estándar son utilizadas para medir la dispersión, como medida del riesgo de la variable aleatoria rentabilidad; ésta medición debe realizarse en forma individual, a cada activo y a todo el portafolio; y c) la conducta racional del inversionista lo lleva a preferir la composición de un portafolio que le represente la mayor rentabilidad, para determinado nivel de riesgo.

La formulación matemática primal del modelo de Markowitz, que se presenta en (1), consiste en determinar las ponderaciones $w_{i}$ que maximizan el rendimiento esperado del portafolio, sujeto a un riesgo máximo admitido. Es decir:

$\operatorname{Max} E(R p)=\sum_{i=1}^{n} w_{i} \cdot E\left(R_{i}\right)$

Sujeto a (2)

$$
\begin{aligned}
& \sigma^{2}\left(R_{p}\right)=\sum_{i=1}^{n} \sum_{j=1}^{n} w_{i} \cdot w_{j} . \sigma_{i j} \leq \sigma_{0}^{2} \\
& \sum_{i=1}^{n} w_{i}=1 ; \quad w_{i} \geq 0(i=1, \ldots, n)
\end{aligned}
$$

donde $\mathrm{n}$ es el número de activos en el portafolio; $R_{i}$ es la variable aleatoria rendimiento del activo $i ; E\left(R_{i}\right)$ es el rendimiento esperado del activo $i ; R_{p}$ es la variable aleatoria rendimiento del portafolio; $E\left(R_{p}\right)$ es el rendimiento esperado del portafolio; $w_{i}$ es la proporción del presupuesto del inversionista destinado al activo $i$; $\sigma^{2}\left(R_{p}\right)$ es la varianza del rendimiento del portafolio; $\sigma_{i j}$ es la covarianza entre los rendimientos de los activos $i$ y $j$; y $\sigma_{0}^{2}$ es la varianza máxima admitida.

La formulación dual alternativa consiste en determinar las ponderaciones que minimizan la varianza del portafolio, sujeto a un rendimiento mínimo requerido para el portafolio. En forma matemática (3): 
$\operatorname{Min} \sigma^{2}\left(R_{p}\right)=\sum_{i=1}^{n} \sum_{j=1}^{n} w_{i} \cdot w_{j} \cdot \sigma_{i j}$

Sujeto a (4)

$$
\begin{aligned}
& E\left(R_{p}\right)=\sum_{i=1}^{n} w_{i} \cdot E\left(R_{i}\right) \geq \mu_{0} \\
& \sum_{i=1}^{n} w_{i}=1 ; \quad w_{i} \geq 0(i=1, \ldots, n)
\end{aligned}
$$

donde $\mu_{0}$ es el rendimiento mínimo requerido.

Con cualquiera de las dos alternativas, optimizando la varianza o el valor esperado, se encuentran las ponderaciones de los activos, que optimizan el objetivo con las restricciones dadas, y se puede determinar un conjunto de portafolios eficientes, que proporcionen el máximo rendimiento para cada nivel de riesgo.

El principal aporte del modelo de Markowitz para la selección de un portafolio óptimo se encuentra en su utilidad para recoger los aspectos fundamentales que deben guiar a un inversionista racional en la elección de la composición de su portafolio, de tal forma, que le produzca la máxima rentabilidad, al controlar el riesgo; o en forma alternativa, minimizar el riesgo, controlando el rendimiento.

El modelo Markowitz, por el cual su autor Harry Markowitz se hizo merecedor al premio Nobel de Economía en 1990, ha sido el punto de partida para que varios investigadores hagan aportes adicionales con el objetivo de lograr un modelo más consistente y eficaz. Sobre ese modelo, académicamente sólido, se han basado gran parte de las metodologías posteriores para la selección y optimización de portafolios.

\subsection{Desventajas del Modelo de Markowitz}

A pesar del gran avance del modelo Markowitz que supuso considerar el portafolio de inversión como un todo, algunos inves- 
tigadores han encontrado serios inconvenientes, entre los cuales sobresalen los siguientes:

Michaud (1989) considera que el uso de series de rentabilidades históricas, en la estimación de los parámetros esperados, produce sesgos importantes. Por ello los portafolios eficientes resultantes en el modelo se componen con activos de alta rentabilidad, reducida varianza y baja correlación con otros activos, de lo que resultan portafolios altamente concentrados en unos pocos títulos (baja diversificación y alto riesgo). Sin embargo, esta dificultad se puede solucionar introduciendo restricciones adicionales que limiten el porcentaje máximo de los recursos que van a ser invertidos en cada título (Michaud, 1989; Haugen, 1993). Para correr el modelo se toman datos históricos; es decir, se supone que el mercado se comportará de forma similar como lo hizo en el pasado, asumiendo estabilidad del mercado, lo cual no siempre es cierto.

Generalmente el gestor piensa directamente en ponderaciones de los activos en el portafolio, no en términos de contribución en rentabilidad / riesgo, ni en predecir vectores de retorno y volatilidad esperados, para cada uno de los activos de su universo de elección. A pesar del reconocimiento académico del modelo, se observa escaso impacto en situaciones reales de estructuración de portafolios. Las soluciones obtenidas por el modelo son poco intuitivas y sorprendentemente inestables dependiendo, sobre todo, de las previsiones sobre las rentabilidades esperadas. Pequeños cambios en las rentabilidades esperadas generan modificaciones muy significativas en el portafolio señalado como óptimo.

En cualquier decisión de inversión, el inversionista se encuentra frente a dos objetivos en conflicto: la ambición de una ganancia máxima y el temor que representa asumir el riesgo para alcanzar esa ganancia. Para cada situación particular tendrá que decidir por una combinación "ganancia-riesgo" que represente y satisfaga sus expectativas.

Black \& Litterman $(1991 ; 1992)$ propusieron un modelo para reducir las dificultades presentadas en el modelo de Markowitz, basado en métodos Bayesianos. El interés de los métodos bayesianos radica básicamente en la posibilidad de incorporar conocimiento extra muestral "a priori" en la estimación de los modelos. La importancia de la propuesta de Black-Litterman radica preci- 
samente en la inclusión de elementos subjetivos e intuitivos, como son las expectativas que tiene el inversionista acerca del rendimiento esperado de un activo. Este modelo se conoce como el modelo Black-Litterman (MBL).

\section{MODELO DE BLACK-LITTERMAN}

Robert Litterman y Fischer Black desarrollaron el modelo de distribución de activos en la gestión de portafolios (asset allocation), conocido como Black- Litterman Global Asset Allocation Model. Fue publicado en Financial Analyst Journal, en Septiembre de 1992. La propuesta de Black-Litterman parte del modelo de Markowitz, en cuya versión primal los inversionistas, dado un capital inicial, maximizan la utilidad esperada, controlando el riesgo.

\subsection{Descripción del Modelo}

El modelo de Black-Litterman (MBL) parte de una situación de equilibrio de mercado, es decir de una serie de rentabilidades esperadas que igualen la oferta y la demanda de activos financieros, si todos los inversionistas tuvieran las mismas expectativas. En el MBL, si las expectativas del inversionista no difieren con respecto a las del mercado, no es necesario especificar un rendimiento para cada activo, ya que éstos entran al modelo con su respectivo retorno de equilibrio. El paso a seguir es la obtención de la rentabilidad esperada que se alcanza por optimización inversa; es decir, en lugar de preguntarse qué ponderación es necesaria para tener determinada rentabilidad, se plantea qué rentabilidad esperada supone la ponderación que indica la capitalización.

Después de calcular la rentabilidad esperada, el modelo procede con uno de sus más importantes aportes, la incorporación de las expectativas que el inversionista tiene del mercado. Una expectativa es una suposición acerca del futuro, y puede o no ser realista. Para el caso de un portafolio de inversión, se refiere a las perspectivas o expectativas sobre la evolución futura de un título o de un sector; además, para cada una se especifica un nivel de confianza, 
que es la probabilidad a priori de que se cumpla esa expectativa, según el inversor.

Las expectativas pueden ser de tres tipos: Absoluta: Por ejemplo, el sector tecnológico tendrá una rentabilidad del 3\%, inferior a la implícita del mercado del 3,73\% (confianza en la visión del 50\%). Relativa simple: El sector energético superará al de telecomunicaciones en un 6\% (confianza en la visión del 60\%). Relativa múltiple: Conjuntamente, el sector comercial y el financiero superarán al industrial y de servicios en un 0,5\% (confianza del 40\%). Así que si la rentabilidad del sector comercial y financiero ponderado es del $4 \%$ frente al $3.2 \%$ de los segundos, se tendría que el exceso es de un $0,8 \%$. La visión lo reduce al $0,5 \%$, por lo cual es una visión negativa sobre el primer par de sectores.

El modelo Black-Litterman como versión mejorada del modelo Markowitz considera los siguientes aspectos: Hay $n$ activos, con capitalizaciones $M_{i}, i=1,2, \ldots n$. La capitalización de mercado es igual al número de títulos o unidades del activo disponibles en el mercado por su respectivo precio. Las ponderaciones de mercado de los $\mathrm{n}$ activos están dadas por el vector $W=\left(W_{1}, W_{2}, \ldots, W_{n}\right)$, en donde la ponderación del activo i es (5):

$W i=\frac{M_{i}}{\sum_{i=1}^{n} M_{i}}$

El coeficiente de aversión al riesgo $(\lambda)$, que es una constante que se determina como (6):

$\lambda=\frac{R_{M}-R_{f}}{\sigma_{M}^{2}}$

donde $R_{M}$ es el retorno del mercado; $R_{f}$ es la tasa libre de riesgo y $\sigma_{M}^{2}$ es la varianza del retorno del mercado. El exceso de retornos implícitos de equilibrio (П) está dado por (7).

$\Pi=\lambda \Sigma W$

Los retornos especificados por (7) se llaman retornos implícitos de equilibrio, debido a que si los precios de los activos se ajustan 
hasta que los retornos esperados sean iguales a lo que consideran los inversionistas, suponiendo que todos tienen la misma expectativa de mercado, esos ajustes hacen que la demanda iguale la oferta. El vector de excesos de retornos $\boldsymbol{R}=\left(R_{1}, R_{2}, \ldots, R_{n}\right)$. Los retornos en exceso son iguales al retorno de cada activo menos la respectiva tasa libre de riesgo. Se supone que el vector de excesos de retornos tiene una distribución normal con retorno esperado $\mu$ y matriz de covarianza $\Sigma$. Es decir (8):

$R \sim N(\mu, \Sigma)$

Se supone que $\mu$ tiene una distribución de probabilidad que es proporcional al producto de dos distribuciones normales. La primera distribución representa el equilibrio (9)

$\mu \sim N(\Pi, \tau \Sigma)$

donde $\tau$ es una constante que refleja el grado de incertidumbre con respecto a la precisión con la que es calculado П. Si el grado de incertidumbre es alto $\tau$ será cercano a cero, en caso contrario, $\tau$ será cercano a uno. $\tau$ se determina con un valor entre cero y uno, generalmente entre 0,01 y 0,05 (Idzorek, 2004), ya que la incertidumbre sobre la media debe ser menor a la incertidumbre de la variable.

La segunda distribución representa las expectativas del inversionista sobre los retornos del mercado. Se tiene un conjunto de $\mathrm{k}$ expectativas representadas con relaciones lineales. La expectativa se plantea como que el retorno esperado de un portafolio $p_{k}$ tiene una distribución normal con promedio $q_{k} \mathrm{y}$ una desviación estándar dada por $\omega_{k}$. Las $\mathrm{k}$ expectativas con los correspondientes retornos esperados se expresan como (10) y (11)

$\mathrm{P}^{\mathrm{T}}=\left[\mathrm{p}_{1}, \mathrm{p}_{2}, \ldots, \mathrm{p}_{k}\right]$

$\mathrm{Q}^{\mathrm{T}}=\left[\mathrm{q}_{1}, \mathrm{q}_{2}, \ldots, \mathrm{q}_{k}\right]$

P es la matriz que selecciona los activos que hacen parte de una expectativa y $\mathrm{Q}$ es el vector de expectativas. Contiene el retorno esperado para cada portafolio $p_{k}$. 
Mediante las expectativas planteadas en (10) y (11), se utiliza un esquema de ponderación por capitalización de mercado para determinar cada uno de los elementos de $\mathrm{P}$ diferentes de cero, en vez de utilizar un esquema de igual ponderación (Idzorek, 2004). Así, la ponderación individual de cada activo es proporcional a la capitalización de mercado del activo dividida por la capitalización del mercado total de los activos con cualquiera que sea su desempeño (positivo o negativo). La manera de expresar las expectativas es la siguiente (12):

$\mathrm{P} * \mu=\mathrm{Q}+\varepsilon$

donde $\mathrm{P}$ es la matriz conocida $\mathrm{K} \mathrm{x} \mathrm{n}$; $\mathrm{Q}$ es el vector de expectativas conocido $\mathrm{K}$ x 1 ; $\varepsilon$ es el vector aleatorio $\mathrm{K}$ x 1 con media cero y matriz diagonal de covarianzas $\Omega$, normalmente distribuido. Entonces (13)

$\mathrm{P} * \mu \sim \mathrm{N}(\mathrm{Q}, \Omega)$

donde $\Omega$ es la matriz diagonal $K x K$ con elementos $\omega_{i i}$ en la diagonal y ceros en el resto de posiciones ya que se considera que las expectativas no están relacionadas. Mientras mayor sea $\omega_{i i}$ significa que existe un grado de confianza menor en los retornos esperados Q.

\subsection{Confianza en las Expectativas: Método de Idzorek}

Idzorek (2004) propone ajustar la matriz Q controlando las desviaciones del portafolio causadas por las expectativas. El método ayuda a encontrar los niveles de confianza implícitos en las expectativas, y éstos pueden utilizarse con un nivel de confianza establecido por el inversionista, el cual está entre 0 y $100 \%$. En esa forma se logra incluir, además de la varianza, otras consideraciones que afectan el nivel de confianza en la o las expectativas.

La propuesta de Idzorek es un modelo lineal que reorganiza la fórmula $\Pi=\lambda \Sigma W$ y reemplaza el vector de retornos de equilibrio $\Pi$ por un vector que representa cualquier conjunto de retornos $\mu$. Entonces (14): 
$W=(\lambda \Sigma)^{-1} \mu$

Si en (14) se reemplaza $\mu$ con el vector de retornos de plena confianza en las expectativas, $\mu_{\mathrm{BL} 100 \%}$, se encuentra $W_{\mathrm{BL} 100 \%}$, el vector de ponderaciones basado en un nivel de confianza del 100\% en las expectativas. Además, si se reemplaza $\mu$ en la fórmula por el vector de retornos de equilibrio $\Pi$, y el vector de retornos de BlackLitterman, $\mu_{\mathrm{BL}}$, se encuentran las ponderaciones de mercado $W_{\text {mcd }}$ y las ponderaciones de Black-Litterman $W_{\mathrm{BL}}$.

Así es posible, según Idzorek, determinar un nivel de confianza implícito en las expectativas dividiendo cada diferencia de ponderaciones $\left(W_{\mathrm{BL}}-W_{\mathrm{mcd}}\right)$, por la correspondiente diferencia máxima $\left(W_{100 \%}-W_{\mathrm{mcd}}\right)$. El nivel de confianza implícito en las expectativas está dado por (15):

Nivel de confianza implícito $=\frac{\left(W_{\mathrm{BL}}-W_{\mathrm{mcd}}\right)}{\left(W_{100 \%}-W_{\mathrm{mcd}}\right)}$

Este vector refleja la varianza del portafolio de acuerdo a cada expectativa, pero no incluye el nivel de confianza que tiene el inversionista en la misma. Para incluir el nivel de confianza del inversionista, Idzorek propone generar desvíos de la siguiente forma (16)

Desvío $_{k} \approx\left(W_{100 \%}-W_{\mathrm{mcd}}\right) * \mathrm{C}_{\mathrm{k}}$

donde Desvío $_{k}$ es la desviación aproximada causada por la késima expectativa, y $\mathrm{C}_{\mathrm{k}}$ es la confianza del inversionista en la késima expectativa. Como no hay otras expectativas, el vector aproximado de ponderaciones recomendadas, resultante de la expectativa $\mathrm{k}$, está dado por (17):

$W_{\mathrm{k}, \%}=W_{\mathrm{mcd}}+$ Desvío $_{k}=W_{\mathrm{mcd}}+\left(W_{100 \%-} W_{\mathrm{mcd}}\right) * \mathrm{C}_{\mathrm{k}}$

donde $W_{\mathrm{k}, \%}$ es el vector de ponderaciones objetivo basado en la desviación causada por la k-ésima expectativa. En este método se 
mantiene constante el escalar $\tau$ y de esta manera no se afecta el vector de retornos.

\subsection{Consistencia de las Expectativas: Método de Fusai y Meucci}

El modelo Black-Litterman es perfeccionado a través de la metodología de Idzorek (2004) que flexibiliza aún más la inclusión de expectativas en el modelo. Adicionalmente, Fusai \& Meucci (2003) plantean una forma de validar la consistencia de las expectativas. Estos autores presentan un método para cuantificar la diferencia estadística entre los retornos previos y los retornos posteriores estimados. En ella se aporta una forma para definir expectativas que contrastan con los rendimientos de equilibrio y controlan la incertidumbre en estas expectativas para hacerlas más consistentes con los retornos previos. Estos autores proponen aplicar la distancia de Mahalanobis para encontrar los retornos esperados de Black-Litterman que se consideran posibles. Esta distancia está dada por (18):

$M_{Q}^{2}=\left(\mu_{\mathrm{BL}}-\Pi\right)^{\mathrm{T}}(\tau \Sigma)^{-1}\left(\mu_{\mathrm{BL}}-\Pi\right)$

Esta distancia tiene una distribución chi cuadrado con $\mathrm{n}$ grados de libertad (n es el número de activos) y puede ser transformada en una probabilidad de que el vector de perspectivas $Q$ sea consistente. Si la distancia es pequeña o está por debajo de un umbral determinado por el decisor, las expectativas no están distantes de los retornos de equilibrio y la consistencia entre los retornos de Black-Litterman y los retornos de equilibrio es alta; de no ser así, el inversionista debe explorar la posibilidad de que alguna de las expectativas planteadas sea muy agresiva y por lo tanto deba ser modificada. Fusai y Meucci proponen calcular el índice de consistencia (19):

$C_{Q}=1-F\left(M_{Q}^{2}\right)$

Donde $F$ es la probabilidad acumulada de la distribución chicuadrado con $\mathrm{n}$ grados de libertad. Cuando el índice de consistencia está por debajo de un valor determinado, el cambio más míni- 
mo afecta el nivel de consistencia, por lo que se deben ajustar las expectativas agresivas. Los mismos autores proponen calcular la sensibilidad del índice de consistencia a cada una de las expectati$\operatorname{vas}(20)$ :

$\frac{\partial C_{Q}}{\partial Q}=\frac{\partial C \partial M^{2} \partial \mu_{B L}}{\partial M^{2} \partial \mu_{B L} \partial Q}=-2 f\left(M_{Q}^{2}\right)\left(P \Sigma P^{T}+\Omega\right)^{-1}-P\left(\mu_{B L}-\Pi\right)$

donde $f$ es la densidad de probabilidad de la distribución chicuadrada con $n$ grados de libertad. De esta manera, se calcula el vector de sensibilidades para buscar la expectativa con el valor absoluto más grande. Si la sensibilidad es positiva, la expectativa $Q(\mathrm{k})$ debe incrementarse, o en caso contrario, reducirse.

De cualquier forma, Black-Litterman arroja resultados sensibles a cambios en el mercado, por lo que se desarrolla una versión modificada que se llama Black-Litterman modificado. En este caso, se incluyen los pesos modificados de acuerdo a las expectativas del mercado. Igualmente se calcula el vector de excesos de retornos de equilibrio, que está dado por: $\Pi=\lambda \Sigma W$. Al resultado no se le llama retornos esperados sino umbrales de rentabilidad mínima exigida a los activos.

Con respecto al vector $\Pi$ se tiene lo siguiente: Si se cumple que $\Pi>$ retorno esperado, es un indicador de demasiado peso asignado. Si por el contrario, $\Pi<$ retorno esperado, indicaría insuficiente peso asignado. Se realizan los ajustes hasta que los retornos implícitos de equilibrio sean consistentes con las expectativas.

Walters (2009) proporciona una minuciosa deducción del modelo de Black-Litterman, partiendo de la publicación original de los autores, sintetiza resultados de diversos artículos publicados sobre el tema y desarrolla aplicaciones completas para cada uno de los aspectos tratados. Además, Cheung (2009) contribuye con un estudio adicional que permite una mejor comprensión del propio modelo a través de una interpretación económica, una aclaración de la formulación y los supuestos del modelo, así como una guía para su implementación. 


\subsection{Ventajas del Modelo Black-Litterman}

La principal ventaja del modelo Black-Litterman frente al modelo de Markowitz es que permite incluir las expectativas del inversor y de acuerdo a la confianza que se maneja sobre las mismas, se da un mayor o menor peso al activo dentro del portafolio. El modelo Black-Litterman es favorable principalmente para administradores que están constantemente buscando buenas estrategias y que estudian y revisan constantemente el comportamiento del mercado.

Se puede interactuar con el modelo de Black-Litterman que permite incluir expectativas y medir la consistencia de las mismas de acuerdo a la propuesta de Fusai y Meuci, para lograr portafolios razonables e intuitivos. El modelo Black-Litterman es flexible, lo cual puede evidenciarse en aspectos como los siguientes: además de incluir las expectativas del inversor sobre los activos que intervienen en la optimización, esas expectativas pueden ser actualizarlas en cualquier momento, sin afectar sustancialmente la estructura del modelo; asimismo, gracias a la metodología Idzorek, el decisor puede asignar diferentes niveles de confianza a las expectativas.

Otra ventaja es que el modelo de Markowitz requiere los retornos esperados de cada activo, mientras que el modelo BlackLitterman acepta que el inversor incluya, entre las expectativas, retornos de los activos sobre los cuales tiene información importante, que considera puede generar impacto positivo o negativo en el portafolio. Este modelo considera que la información histórica no es suficientemente confiable para tomar decisiones frente a la asignación estratégica de activos en un portafolio. En cambio, reconoce la importancia de las expectativas y la confianza que se tenga en ellas. El modelo le da la posibilidad al inversor de asumir un riesgo alto o bajo para un activo determinado, dependiendo de la confianza que tenga sobre la expectativa correspondiente a ese activo. También diferencia entre la magnitud de la expectativa y el grado de certeza que se tiene sobre la misma.

Al utilizar los retornos de equilibrio como eje central para el modelo Black-Litterman, se logran portafolios equilibrados y estables en el tiempo. Es decir, que las posiciones estratégicas pueden 
mantenerse por lo menos durante el período de tiempo para el cual están planteadas las expectativas, lo que disminuye la necesidad de una reestructuración continua.

\subsection{Extensiones del Modelo Black-Litterman}

La metodología Black-Litterman no produce por si sola estrategias de inversión. Es necesario incorporar un conjunto de expectativas, y realizar simulaciones para evaluar la consistencia de las expectativas, como lo plantean Fusai y Meucci. En este contexto, cada simulación no sólo constituye una prueba sobre el modelo, sino también una prueba sobre la estrategia que producen las expectativas.

El modelo Black-Litterman parte del supuesto de normalidad, lo que no siempre suele darse en los mercados. Múltiples estudios muestran que existe una gran diferencia entre las distribuciones empíricas de los rendimientos y la distribución normal; la distribución de los rendimientos tiende a ser más picuda o leptocúrtica que la distribución normal y posee colas más pesadas, lo que implica que existe mayor probabilidad de obtener valores extremos que la que existiría en una distribución normal.

Siempre será viable que se desarrolle un modelo que busque minimizar las debilidades que presenta el anterior, tratando de lograr la mejor optimización de los portafolios. En ese sentido, Herold (2003) agrega un elemento al modelo de Black-Litterman, mediante una metodología que permite incorporar expectativas de manera cualitativa, lo cual hace posible un mayor equilibrio en los portafolios, en términos de perfil de riesgo.

Meucci (2005) extiende la metodología Black-Litterman a distribuciones genéricas en mercados no normales y a expectativas no normales. Adicionalmente, Giacometti et al. (2005) complementan el modelo clásico de Black-Litterman mediante la aplicación de modelos más realistas para el rendimiento de activos (la t de Student, y las distribuciones estables) y mediante el uso de medidas alternativas de riesgo tales como la dispersión, el valor en riesgo y el valor en riesgo condicional. Además, ellos encuentran que la inclusión de las visiones del inversionista en el modelo, proporcio- 
na información acerca de cómo las diferentes hipótesis distribucionales pueden impactar la composición óptima del portafolio.

Martellini \& Ziemann (2007) presentan una extensión del modelo bayesiano de Black-Litterman en la que incluyen preferencias no triviales sobre los momentos de orden superior (sesgo y curtosis) de la distribución de retornos de los activos. Además, consideran la no normalidad y la incertidumbre de los parámetros de esa distribución.

Cheung (2008) y Meucci (2008) exponen cómo se pueden utilizar modelos factoriales de proyección de retornos junto con el MBL. Martellini \& Ziemann (2010) desarrollan estimadores mejorados para los parámetros cosesgo y cocurtosis incluidos en su extensión de Black-Litterman desarrollada en el 2007. A nivel mundial se han desarrollado diversas aplicaciones que permiten resaltar las ventajas del modelo BL y sus extensiones con respecto al modelo clásico de Markowitz.

Jaureguízar (2007), desarrolla una aplicación del MBL con base en los 18 sectores de la bolsa europea representados en los 18 subíndices del EuroStoxx 50, y concluye que el MBL permite generar estructuras de portafolios que pueden utilizarse en la práctica, ya que soluciona el problema del punto de partida de las rentabilidades esperadas por medio del equilibrio de mercado. Además permite incorporar las visiones del gestor de manera eficiente, cuantitativa y coherente. Así mismo, se hace evidente que el gestor puede complementar el MBL mediante extensiones más sofisticadas de estimación de volatilidades y covarianzas.

Martínez (2009), al aplicar el MBL a un portafolio del Banco Central de Bolivia, destaca los beneficios que se obtienen en términos de diversificación y flexibilidad, respecto al modelo tradicional. En el mercado colombiano, Trujillo (2009) desarrolla una minuciosa aplicación del MBL a fondos de pensiones colombianos, y muestra que a diferencia del método de Markowitz, el MBL proporciona portafolios más diversificados e intuitivos, por lo que se constituye en una importante herramienta para la toma de decisiones de inversión. 


\section{CONCLUSIONES}

El modelo de Markowitz como referente teórico en la optimización de portafolios es de gran utilidad para los analistas y gestores de inversiones, ya que ha proporcionado portafolios con mejor desempeño que los índices de referencia del mercado; sin embargo, cabe aclarar que el éxito en su aplicación depende de la correcta estimación de los rendimientos esperados de los títulos y de sus covarianzas. Además, tampoco se puede olvidar que sus cálculos se realizan tomando series de rentabilidades históricas, las cuales no permiten asegurar que el comportamiento futuro del mercado sea similar a como fue en el pasado.

En la práctica el modelo de Black-Litterman es más consistente en el proceso de asignación de activos que el modelo de Markowitz; como punto de partida soluciona el problema del cálculo de las rentabilidades esperadas por medio del portafolio que proporciona el equilibrio del mercado. Es más flexible y proporciona mayor posibilidad de diversificación al permitir, en primera instancia, la inclusión o no de expectativas que se tengan de cada activo componente del portafolio; además posibilita valorar esas expectativas mediante la metodología de Idzorek.

Finalmente cabe resaltar la importancia de tener una metodología que haga posible obtener resultados diversificados y que admita a los gestores, en la práctica, orientar la asignación estratégica del capital de inversión de acuerdo a las visiones o expectativas que se tengan sobre los mercados financieros globales, y éstas son características relevantes del modelo Black-Litterman y sus extensiones.

\section{REFERENCIAS}

Black, F., Litterman, R., (1991); Asset allocation: Combining investor views with market equilibrium, Goldman, Sachs \& Co., Fixed Income Research.

Black, F., Litterman R., (1992); Global portfolio optimization, Financial Analysts Journal, 48(5), 28-43. 
Cheung, W., (2009); The Black-Litterman model explained, Working paper, Available at SSRN: http://ssrn.com/abstract=1312664.

Giacometti, R., Bertocchi, M., Rachev, S., Fabozzi, F., (2005); Stable distributions in the Black-Litterman approach to the asset allocation, Working paper.

Haugen, R.A., (1993); Modern investment theory, Prentice Hall, Englewood Cliffs, New Jersey, 3a ed.

Herold, U., (2003); Portfolio construction with qualitative forecasts, The Journal of Portfolio Management, Fall, 61-72.

Idzorek, T., (2004); A step-by-step guide to the Black-Litterman model: incorporating user specified confidence levels, Zephyr Associates, Inc.

Jaureguízar, C. (2007); Black-Litterman global asset allocation model, Noesis Análisis Financiero, Quantitative Research Division.

Martellini, L., Ziemann, V., (2007); Extending Black-Litterman analysis beyond the mean-variance framework, The Journal of Portfolio Management, 33(4), 33-44.

Martellini, L., Ziemann, V., (2010); Improved estimates of higher-order comoments and implications for portfolio selection, Rev. Financ. Stud., 23, 1467-1502.

Martinez, O., (2009); Aplicación del modelo Black Litterman a la optimización de portafolios del BCB, Banco central de Bolivia.

Markowitz, H., (1952); Portfolio selection, Journal of Finance, 7(1), 77-91.

Meucci, A., (2005); Risk and asset allocation, Springer, New York.

Meucci, A., (2008); The Black-Litterman approach: original model and extensions, Bloomberg ALPHA research \& Education Paper, No. 2008-01.

Michaud, R., (1989); The Markowitz optimization enigma: is optimized optimal?, Financial Analysts Journal, 45(1), 31-42.

Trujillo, M.E., (2009); Construcción y gestión de portafolios con el modelo Black-Litterman: Una aplicación a los fondos de pensiones obligatorias en Colombia, Trabajo de grado, Universidad de los Andes.

Walters, J., (2009); The Black-Litterman model in detail, Working paper, Available at SSRN: http://ssrn.com/abstract=1314585. 